Алгебра и анализ

Том. 15 (2003), вып. 6
St. Petersburg Math. J.

Vol. 15 (2004), No. 6, Pages 867-874

S 1061-0022(04)00836-2

Article electronically published on November 16, 2004

\title{
SOME GEOMETRIC PROPERTIES OF CONVEX BODIES. II
}

\author{
V. V. MAKEEV
}

\begin{abstract}
Topological means are used for the study of approximation of 2-dimensional sections of a 3-dimensional convex body by affine-regular pentagons and approximation of a centrally symmetric convex body by a prism. Also, the problem of estimating the relative surface area of the sphere in a normed 3-space, the problem on universal covers for sets of unit diameter in Euclidean space, and some related questions are considered.
\end{abstract}

Throughout, by a convex body $K \subset \mathbb{R}^{n}$ (a figure for $n=2$ ) we mean a compact convex subset of $\mathbb{R}^{n}$ with nonempty interior.

We denote by $G_{k}\left(\mathbb{R}^{n}\right)$ (respectively, $G_{k}^{+}\left(\mathbb{R}^{n}\right)$ ) the Grassmann manifold of nonoriented (respectively, oriented) $k$-planes in $\mathbb{R}^{n}$ passing through $O \in \mathbb{R}^{n}$. We let

$$
\gamma_{k}^{n}: E_{k}\left(\mathbb{R}^{n}\right) \rightarrow G_{k}\left(\mathbb{R}^{n}\right) \quad \text { and } \quad\left(\gamma_{k}^{n}\right)^{+}: E_{k}^{+}\left(\mathbb{R}^{n}\right) \rightarrow G_{k}^{+}\left(\mathbb{R}^{n}\right)
$$

be the tautological fiber bundles, where the fiber over an (oriented) $k$-plane $\alpha \in G_{k}\left(\mathbb{R}^{n}\right)$ is $\alpha$ itself regarded as a $k$-dimensional vector space.

We say that a field of convex bodies (or figures; a $C B$ - or a $C F$-field) is given in a vector bundle $\gamma$ if in each fiber $\alpha$ of $\gamma$ we mark a convex body $K(\alpha)$ depending continuously on $\alpha$. A CB-field is pointed if for each $\alpha$ we also mark a point $x(\alpha) \in K(\alpha)$ depending continuously on $\alpha$. (In other words, $x(\alpha)$ is a section of $\gamma$.)

If $\lambda \in \mathbb{R}$ and $P$ is an affine-regular polygon (e.g., a pentagon or a parallelogram) with center $O(P)$, then $\lambda P$ denotes the polygon homothetic to $P$ with homothety ratio $\lambda$ and homothety center $O(P)$.

We denote by $S(K)$ the area of a figure $K \subset \mathbb{R}^{2}$.

\section{$\S 1$. FiELDS OF CONVEX FIGURES IN $\gamma_{2}^{3}$ AND $\left(\gamma_{2}^{3}\right)^{+}$, AND 2-DIMENSIONAL SECTIONS OF CONVEX BODIES IN $\mathbb{R}^{3}$}

First, we prove two corollaries to the following known result.

Theorem [2]. Each CF-field in $\gamma_{2}^{3}$ contains a figure circumscribed about an affine-regular octagon.

Corollary 1. Suppose $C$ is the bounded component of a cubic surface in $\mathbb{R}^{3}$. Then each inner point $O$ of $C$ lies in a plane intersecting $C$ along an ellipse.

Proof. Indeed, $C$ is convex automatically, because otherwise $C$ intersects some line at 4 points. Consequently, the section of $C$ by some 2-plane through $O$ is circumscribed about an affine-regular octagon. Then this section is a component of a cubic, intersects an ellipse at 8 points, and, consequently, is an ellipse by the Bézout theorem.

2000 Mathematics Subject Classification. Primary 52A10, 52A15.

Key words and phrases. Convex body, figure, field of convex bodies, relative surface area.

The paper was revised by the author for the English edition. 
Remark. Certainly, this follows from standard facts of algebraic geometry: any plane that passes through a point inside $C$ and a real line lying on the cubic surface intersects $C$ along an ellipse. But actually we have proved more: each field of bounded components of cubics in $\gamma_{2}^{3}$ contains an ellipse.

Corollary 2. Each CF-field in $\gamma_{2}^{3}$ contains a figure $K$ containing a parallelogram $P$ such that

$$
P \subset K \subset\left(1+\frac{\sqrt{2}}{2}\right) P .
$$

Proof. Indeed, the figure $K$ circumscribed about an affine-regular octagon $\Omega=A_{1} \ldots A_{8}$ possesses the required property. In this case, $K$ is contained in the octagonal star $\Sigma$ bounded by segments of the rays that extend the sides of $\Omega$. Letting $P$ be the parallelogram $A_{2} A_{4} A_{6} A_{8}$, we easily see that $P \subset K \subset \Sigma \subset\left(1+\frac{\sqrt{2}}{2}\right) P$.

Remark. Considering fields of disks, we see that $1+\frac{\sqrt{2}}{2}$ cannot be replaced by a constant smaller than $\sqrt{2}$.

Theorem 1. Each pointed CF-field $(K(\alpha), x(\alpha))$ in $\left(\gamma_{2}^{3}\right)^{+}$contains a figure $K(\alpha)$ circumscribed about an affine-regular pentagon with center at the marked point $x(\alpha)$.

Corollary 3. Each field of centrally symmetric convex figures in $\left(\gamma_{2}^{3}\right)^{+}$or $\gamma_{2}^{3}$ contains a figure circumscribed about an affine-regular decagon.

Corollary 4. If a field of centrally symmetric convex figures in $\left(\gamma_{2}^{3}\right)^{+}$consists of bounded components of curves of degree at most 4 , then the field contains an ellipse.

Indeed, by the Bézout theorem, the figure circumscribed about an affine-regular decagon is an ellipse.

Corollary 5. Each convex bounded centrally symmetric quartic in $\mathbb{R}^{3}$ has a planar central section that is an ellipse.

The proof of Theorem 1 uses two topological lemmas.

Lemma 1. Suppose $n \in \mathbb{N}, W$ is a compact oriented $2 n$-manifold, the cyclic group $\mathbb{Z}_{2 n+1}$ freely acts on $W$, and the boundary $\partial W$ of $W$ with standard orientation is the union of two closed $\mathbb{Z}_{2 n+1}$-invariant manifolds $M$ and $M^{\prime}$. We let $\mathbb{Z}_{2 n+1}$ act on $\mathbb{R}^{2 n+1}$ by cyclic permutations of coordinates of points and denote by $l \subset \mathbb{R}^{2 n+1}$ the line determined by the equations $x_{1}=x_{2}=\cdots=x_{2 n+1}$.

Suppose that $\mathcal{F}: W \rightarrow \mathbb{R}^{2 n+1}$ is a continuous $\mathbb{Z}_{2 n+1}$-equivariant mapping such that $\mathcal{F}(M)$ and $\mathcal{F}\left(M^{\prime}\right)$ do not intersect $l$. Then

$$
\operatorname{deg}\left(\mathcal{F} \mid: M \rightarrow \mathbb{R}^{2 n+1} \backslash l\right)+\operatorname{deg}\left(\mathcal{F} \mid: M^{\prime} \rightarrow \mathbb{R}^{2 n+1} \backslash l\right) \equiv 0 \quad \bmod 2 n+1 .
$$

Proof. After a slight perturbation, we can assume that $\mathcal{F}$ is smooth and transversal to $l$. Then, $\mathcal{F}^{-1}(l)$ consists of a finite number of orbits of points of $W$. We surround the points by small balls $D_{1}, \ldots, D_{N}$ such that they are mutually disjoint and disjoint from $M$ and $M^{\prime}$ and, furthermore, the balls with centers in one $\mathbb{Z}_{2 n+1}$-orbit are mapped to one another under the action of $\mathbb{Z}_{2 n+1}$.

The mapping $\mathcal{F}$ takes the $2 n$-manifold $\hat{W}:=W \backslash \bigcup_{i=1}^{N} \stackrel{\circ}{D}_{i}$ with boundary to $\mathbb{R}^{2 n+1} \backslash l \simeq$ $S^{2 n-1}$. Consequently, $\operatorname{deg}\left(\mathcal{F} \mid: \partial \hat{W} \rightarrow \mathbb{R}^{2 n+1} \backslash l\right)=0$. We have

$$
\partial \hat{W}=M \cup M^{\prime} \cup \bigcup_{i=1}^{N} \partial D_{i} .
$$

By construction, the $\mathbb{Z}_{2 n+1}$-equivariance of $\mathcal{F}$ implies that $\operatorname{deg}\left(\mathcal{F} \mid: \bigcup_{i=1}^{N} \partial D_{i} \rightarrow \mathbb{R}^{2 n+1} \backslash l\right)$ is divisible by $2 n+1$, which completes the proof. 
Remark. For prime $2 n+1$, Stiefel manifolds yield interesting examples in which the degree under consideration is nonzero (see [1] and below). The author does not know whether this is possible in the case where $2 n+1$ is composite.

We denote by $S O(3)$ the group of orientation-preserving rotations of $\mathbb{R}^{3}$ about some point.

Lemma 2. Suppose $W$ is a compact 4-manifold, the cyclic group $\mathbb{Z}_{5}$ acts freely on $W$, and the boundary $\partial W$ of $W$ with standard orientation is the union of two closed $\mathbb{Z}_{5}$ invariant 3-manifolds $M$ and $M^{\prime}$, where $M^{\prime}$ is $S O(3)$ with standard action of $\mathbb{Z}_{5}$. We let $\mathbb{Z}_{5}$ act on $\mathbb{R}^{5}$ by cyclic permutations of the coordinates of points.

Suppose that $\mathcal{F}: W \rightarrow \mathbb{R}^{5}$ is a continuous $\mathbb{Z}_{5}$-equivariant mapping. Then $\mathcal{F}(M)$ contains a point of the form $(x, x, x, x, y)$, where $y \leq x$ (or, optionally, $y \geq x$ ).

Proof. Let $l \subset \mathbb{R}^{5}$ be the line determined by the equations $x_{1}=x_{2}=\cdots=x_{5}$. If $F(M) \cap l \neq \varnothing$, then we are done. Otherwise, it suffices to prove the following assertion (see [1]).

Assertion. If $F(M) \cap l=\varnothing$, then the degree of the mapping

$$
F \mid: M \rightarrow \mathbb{R}^{5} \backslash l \simeq S^{3}
$$

is not divisible by 5 .

Proof. After a slight perturbation, we can assume that the mapping $F^{\prime}: M^{\prime} \rightarrow \mathbb{R}^{5}$ is a $\mathbb{Z}_{5}$-equivariant mapping with image in $\mathbb{R}^{5} \backslash l$. It is well known that in this case $\operatorname{deg}\left(F^{\prime} \mid: M^{\prime} \rightarrow \mathbb{R}^{5} \backslash l\right)$ is not divisible by 5 (see [1]; actually, $\operatorname{deg}\left(F^{\prime} \mid\right)=-1$ ). Now, the required result follows from Lemma 1.

Proof of Theorem 1. We consider positively oriented affine-regular pentagons inscribed in the figures $K(\alpha)$. If $K(\alpha)$ is a generic smooth field of smooth convex figures in $\left(\gamma_{2}^{3}\right)^{+}$, then the pentagons constitute a compact oriented smooth 3-manifold $M$, on which the cyclic group $\mathbb{Z}_{5}$ acts by cyclic permutations of the vertices of the pentagons.

If all figures $K(\alpha)$ are $\left(C^{1}\right.$-close to) disks, then, obviously, $M \cong S O(3)$.

We define a continuous mapping

$$
F: M \rightarrow \mathbb{R}^{5}
$$

as follows. If $P \subset \alpha$ is a pentagon $A_{1} \ldots A_{5}$ inscribed in $K(\alpha)$ and with center $O(P)$, then the $i$ th coordinate of $F(P)$ is the orthogonal projection of $x(\alpha)$ to the oriented axis $O(P) A_{i}$ with origin at $O(P), i=1, \ldots, 5$.

By construction, $F$ is $\mathbb{Z}_{5}$-equivariant if $\mathbb{Z}_{5}$ acts on $\mathbb{R}^{5}$ by cyclic permutations of the coordinates of points.

Using a smooth generic deformation $K_{t}(\alpha), t \in[0,1]$, we deform the initial field $K_{0}(\alpha):=K(\alpha)$ into a field of figures $K_{1}(\alpha)$ close to disks. Then the oriented affineregular pentagons inscribed in the figures $K_{t}(\alpha)$ form a cobordism between $M$ and $M^{\prime} \cong$ $S O(3)$. By Lemma 2, $F(M)$ contains a point $F(P)=(x, x, x, x, y)$. Simple geometric arguments show that $x=0$, whence $O(P)=x(\alpha)$.

Theorem 2. Each CF-field $K(\alpha)$ in $\left(\gamma_{2}^{3}\right)^{+}$contains a convex figure $K$ circumscribed about an affine-regular pentagon $P$ and such that

$$
P \subset K \subset\left(1+\frac{1}{2 \sin 54^{\circ}}\right) P \subset 1.6181 P .
$$

This estimate is sharp for the field consisting of the sections of a tetrahedron $T$ that pass through an inner point of $T$. 


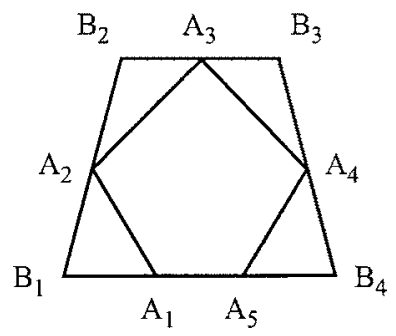

FiguRE 1.

Proof. If $P$ is a pentagon $A_{1} \ldots A_{5}$ inscribed in $K(\alpha)$, then we draw the support lines of $K(\alpha)$ parallel to $A_{1} A_{2}, A_{2} A_{3}, \ldots, A_{5} A_{1}$ and denote by $B_{1}, \ldots, B_{5}$ the points where these support lines touch $K(\alpha)$. We define

$$
F(P):=\left(S\left(\triangle A_{1} A_{2} B_{1}\right), \ldots, S\left(\triangle A_{5} A_{1} B_{5}\right)\right) .
$$

As in the proof of Theorem 1, we see that there is a 2-plane $\alpha$ such that for a certain pentagon $P$ inscribed in $K(\alpha)$ we have

$$
f(P)=(x, x, x, x, y),
$$

where $y \leq x$. We show that $P$ is the required pentagon.

After an affine transformation, we may assume that $P=A_{1} \ldots A_{5}$ is a regular pentagon. Obviously, the altitude of the triangles $\triangle A_{1} A_{2} B_{1}, \ldots, \triangle A_{4} A_{5} B_{4}$ is maximal possible if $S\left(\triangle A_{5} A_{1} B_{5}\right)=0$, and the figure $K(\alpha)$ circumscribed about $P$ is the isosceles trapezoid shown in Figure 1.

Simple calculations show that in this case we have $(*)$.

Remark. From [11] it follows that each CF-field in $\left(\gamma_{2}^{3}\right)^{+}$contains a figure $K$ circumscribed about a regular pentagon $P$, in which case we have

$$
P \subset K \subset(1+\sqrt{5}) P .
$$

(Certainly, the same is true for any affine-regular pentagon inscribed in $K$.) In the general case, the constant $1+\sqrt{5}=1+\tan (\pi / 5) \cot (\pi / 10)$ here cannot be made smaller. Indeed, suppose that the point $O$ lies near the apex of a regular triangular pyramid $T$ the lateral edge of which is many times longer than the edge of the base. We consider the CFfield in $\left(\gamma_{2}^{3}\right)^{+}$consisting of sections of $T$ by planes through $O$. In this case, the sections intersecting the base of $T$ are very prolate, while for the other triangular sections the above constant, obviously, cannot be improved, because two sides of a pentagon inscribed into a triangle lie on the sides of the triangle.

If in Theorem 2 and in the situation considered above we lift the condition that the pentagon $P$ is inscribed, then the sharp values of the constants are not known to the author.

\section{$\S 2$. The Relative surface area of the sphere in a nORMed 3-SPaCe}

Definition. Suppose $P$ is a polyhedron in a finite-dimensional normed space with unit ball $K$. For each hyperface $F$ of $P$, we take the ratio of the area of $F$ and the area of the central section of $K$ parallel to $F$. The sum of these ratios over all hyperfaces of $P$ is the relative surface area of $P$.

In [10], the author constructed a one-parameter family of affine images of a cubeoctahedron which are inscribed in the unit ball of a normed 3-space and have relative surface area of at least $5 / 2$. 


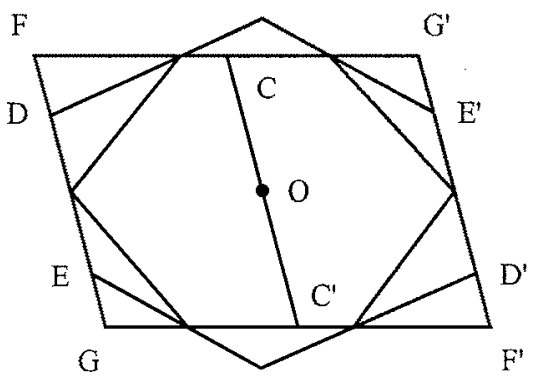

FIGURE 2.

To obtain an upper estimate, we approximate the ball of unit diameter in a normed 3 -space by a circumscribed hexagonal prism.

We need the following result.

Theorem [10]. Suppose $\Pi=A_{1} \ldots A_{12}$ is a regular hexagonal prism, and $A_{13}$ and $A_{14}$ are points that lie outside $\Pi$ on the symmetry axis and are symmetric to each other with respect to the center. Each centrally symmetric convex body $K \subset \mathbb{R}^{3}$ centered at $O$ is circumscribed about an affine image of the 14-tope $P_{14}=A_{1} \ldots A_{14}$ with the same center and such that the parallel support planes of $K$ at the images of $A_{13}$ and $A_{14}$ are parallel to the images of the base planes of $\Pi$.

Corollary 6. Each centrally symmetric convex body $K \subset \mathbb{R}^{3}$ is circumscribed about an affine-regular hexagonal bipyramid $A_{1} \ldots A_{8}$ with the same center and such that the support planes of $K$ at $A_{7}$ and $A_{8}$ are parallel to the plane of the base $A_{1} \ldots A_{6}$, while the support planes of $K$ at $A_{1}, \ldots, A_{6}$ are parallel to the axis $A_{7} A_{8}$ of the bipyramid.

Proof. We apply the above theorem. As the lateral edge of $\Pi$ in $P_{14}$ tends to zero while the length of $A_{13} A_{14}$ remains constant, in the limit we obtain the required bipyramid.

Theorem 3. The unit ball $K$ in a normed 3-space is inscribed in a centrally symmetric hexagonal prism with relative surface area not exceeding $\frac{32}{3}$.

Corollary 7. The relative surface area of $K$ is at most $32 / 3$.

Remark. If $K$ is a Euclidean ball, then the regular prism is a hexagonal prism circumscribed about $K$ with minimal relative surface area, which is equal to $12 \sqrt{3} / \pi$.

The proof involves the following lemma.

Lemma 2. Suppose that the unit disk in a 2-dimensional normed space is an affineregular hexagon $H$. Then the area of any centrally symmetric convex figure $K$ circumscribed about $H$ is at most $\frac{4}{3} S(H)$, and the perimeter of $K$ (with respect to the norm) is at most 8 . Both estimates are sharp.

Proof. Drawing the support lines of $K$ at the vertices of $H$, we reduce the proof to the case where $K$ is a centrally symmetric hexagon.

1) Simple variational arguments show that the area of $K$ is maximal if the vertices of $H$ are the midpoints of the sides of $K$, or $K$ is a parallelogram. In both cases, we have $S(K)=\frac{4}{3} S(H)$.

2) Let $E D$ and $E^{\prime} D^{\prime}$ be a pair of parallel sides of $K$, and let $C C^{\prime}$ be the diameter of $H$ parallel to them (see Figure 2). Continuing the opposite sides $A B$ and $A^{\prime} B^{\prime}$ that contain (respectively) $C$ and $C^{\prime}$, to the intersection with the lines $E D$ and $E^{\prime} D^{\prime}$, we obtain a 
parallelogram $F G F^{\prime} G^{\prime}$ circumscribed about $H$. Obviously,

$$
\frac{E D+E^{\prime} D^{\prime}}{O C}=8 \frac{S(O E D)+S\left(O E^{\prime} D^{\prime}\right)}{S\left(F G F^{\prime} G^{\prime}\right)} \leq 8 \frac{S(O E D)+S\left(O E^{\prime} D^{\prime}\right)}{S(K)} .
$$

Writing this inequality for each pair of parallel sides of $K$ and summing up, we obtain the assertion of the lemma concerning the perimeter of $K$.

Remark. If in the lemma we do not assume the central symmetry of $K$, then it is well known that $S(K) \leq \frac{3}{2} S(H)$, and arguments similar to ours show that the perimeter of $K$ is at most 9 .

Proof of Theorem 3. We assume that $K$ is smooth. In the general case, the result is obtained by passage to the limit.

Let $A_{1} \ldots A_{8}$ be the affine-regular hexagonal bipyramid constructed in Corollary 6 . Obviously, the support planes of $K$ at $A_{1}, \ldots, A_{8}$ bound a hexagonal prism $\Pi$ with centrally symmetric base.

1) The relative areas of the bases of $\Pi$ are at most $4 / 3$ by Lemma 2 , because they are circumscribed about the affine-regular hexagon $A_{1} \ldots A_{6}$ with unit side, which is inscribed in the central section of $K$ parallel to the bases.

2 ) Suppose $P$ is one of the lateral faces of $\Pi$. Then the area of the parallelogram $P$ does not exceed the length of the base of $P$. (Indeed, the area of the central section of $K$ parallel to $P$ cannot be smaller than the area of the parallelogram $P^{\prime}$ having the same directions of sides and such that the lateral side of $P^{\prime}$ is equal to that of $P$, while the base of $P$ is a unit radius of the central section.)

Thus, by Lemma 2, the relative area of the lateral surface of $\Pi$ is at most 8 , and, consequently, the complete relative surface area of $\Pi$ is at most $8+2 \cdot \frac{4}{3}=\frac{32}{3}$.

Theorem 4. Each convex body $K \subset \mathbb{R}^{3}$ is circumscribed about an affine-regular hexagonal bipyramid $A_{1} \ldots A_{8}$ such that the support planes of $K$ at $A_{7}$ and $A_{8}$ are parallel to the plane of the base $A_{1} \ldots A_{6}$.

Proof. We prove this theorem for strictly convex smooth bodies $K$; in the general case, the theorem is obtained by passage to the limit.

For $\alpha \in G_{2}\left(\mathbb{R}^{3}\right)$, we draw the support planes $\alpha_{1}$ and $\alpha_{2}$ of $K$ parallel to $\alpha$, and also the secant plane $\alpha_{3}$ equidistant from $\alpha_{1}$ and $\alpha_{2}$ and parallel to them. We join the points of tangency of $\alpha_{1}$ and $\alpha_{2}$ with $K$ by a segment $I$ and denote by $A(\alpha)$ the orthogonal projection of the point $I \cap \alpha_{3}$ to the plane $\alpha$. Let $B(\alpha)$ be the orthogonal projection to $\alpha$ of the set of the centers of the affine-regular hexagons inscribed in $\alpha_{3} \cap K$.

It suffices to prove that for some $\alpha \in G_{2}\left(\mathbb{R}^{3}\right)$ we have $A(\alpha) \in B(\alpha)$. By construction,

$$
C_{1}:=\left\{A(\alpha) \mid \alpha \in G_{2}\left(\mathbb{R}^{3}\right)\right\}
$$

is the image of a section of $\gamma_{2}^{3}$ that realizes the generator of $H_{2}\left(E_{2}\left(\mathbb{R}^{3}\right) ; \mathbb{Z}_{2}\right)$.

We denote by $\Omega$ the 8-manifold of affine-regular hexagons lying in the planes $\alpha_{3}$. If $K$ is generic, then the affine-regular hexagons inscribed into all possible sections $\alpha_{3} \cap K$ constitute a compact smooth 2 -manifold $\mathcal{H}$ in $\Omega$. Then

$$
C_{2}:=\bigcup\left\{B(\alpha) \mid \alpha \in G_{2}\left(\mathbb{R}^{3}\right)\right\}
$$

is a continuous image of $\mathcal{H}$ that intersects a generic fiber $\alpha \in E_{2}\left(\mathbb{R}^{3}\right)$ at an odd number of points, because a generic convex figure is circumscribed about an odd number of affine-regular hexagons (see [2]). Thus, $C_{2}$ also realizes the generator of $H_{2}\left(E_{2}\left(\mathbb{R}^{3}\right) ; \mathbb{Z}_{2}\right)$. Consequently, the $\mathbb{Z}_{2}$ intersection number of $C_{1}$ and $C_{2}$ is equal to 1 , whence $C_{1} \cap C_{2} \neq$ $\varnothing$. 
Remark. It is easily seen that the volume of any inscribed affine-regular bipyramid in Theorem 4 is at least $\operatorname{Vol}(K) / 6$.

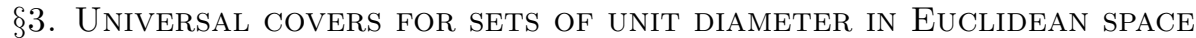

Definitions and examples. A subset $A \subset \mathbb{R}^{n}$ is a universal cover for sets of unit diameter if each subset of $\mathbb{R}^{n}$ of diameter not exceeding 1 is contained in a congruent image of $A$.

A universal cover $A$ is rigid if the set of bodies of constant unit width and such that each of them is contained in finitely many congruent images of $A$ is dense in the Hausdorff metric.

Rigid covers are well known in dimensions 2 and 3. Each centrally symmetric hexagon of unit width is a rigid cover in dimension 2. Borsuk's solution of the Borsuk problem in the plane involved the cover having the form of a regular hexagon of unit width (see [3).

A regular rhombo-dodecahedron of unit width is a rigid cover in 3-space (see [47]). The one-parameter families of rigid covers constructed in 7, 8] consist of centrally symmetric dodecahedra circumscribed about a ball of unit diameter. Is it true that each universal cover contains a rigid universal cover?

A universal cover $A$ is an $s$-cover if there is a $C^{1}$ open and dense set of smooth bodies having constant unit width and such that each of them is contained in an odd number of congruent images of $A$.

By definition, all $s$-covers are rigid. All rigid covers mentioned above are $s$-covers. For $n \geq 3$, the author knows no examples of rigid covers that are not $s$-covers.

The following theorem yields infinite series of $s$-covers in Euclidean spaces.

Theorem 5. Suppose that $A_{n} \subset \mathbb{R}^{n}$ is a centrally and mirror-symmetric s-cover bounded by a finite number of regular hypersurfaces. Let $\Pi$ be the right prism in $\mathbb{R}^{n+1}$ with base $A_{n}$ and unit hight, and let $A_{n+1}$ denote the intersection of $\Pi$ with two unit balls centered at the centers of the bases of $\Pi$. Then $A_{n+1}$ is an s-cover in $\mathbb{R}^{n+1}$.

Proof. Suppose that $K \subset \mathbb{R}^{n+1}$ is a generic smooth body of constant unit width. In the total space $E_{n}\left(\mathbb{R}^{n+1}\right)$ of $\gamma_{n}^{n+1}$, we construct two $n$-dimensional cycles $C_{1}$ and $C_{2}$ intersecting the generic fiber at an odd number of points.

For $\alpha \in G_{n}\left(\mathbb{R}^{n+1}\right)$, we denote by $s(\alpha)$ the point of intersection of $\alpha$ with the line containing the diameter of $K$ perpendicular to $\alpha$. Obviously, $s$ is a section of $\gamma_{n}^{n+1}$, and its image $C_{1}=s\left(G_{n}\left(\mathbb{R}^{n+1}\right)\right)$ intersects each fiber at a unique point.

Consider the fiber bundle $\xi: E(\xi) \rightarrow G_{n}\left(\mathbb{R}^{n+1}\right)$ such that the fiber over a hyperplane $\alpha \in G_{n}\left(\mathbb{R}^{n+1}\right)$ is the set of the congruent images of $A_{n}$ that lie in $\alpha$. (By the mirror symmetry of $A_{n}$, no problems with orientation arise.) For $\alpha \in G_{n}\left(\mathbb{R}^{n+1}\right)$, let $B_{\alpha}$ be the set of the congruent images of $A_{n}$ that contain the orthogonal projection of $K$ to $\alpha$, and let $B=\bigcup_{\alpha} B_{\alpha} \subset E(\xi)$. If $K$ is a generic smooth body, then $B$ is a smooth compact $n$-manifold in $E(\xi)$ intersecting the generic fiber at an odd number of points.

We consider the fiberwise mapping $p: E(\xi) \rightarrow E_{n}\left(\mathbb{R}^{n+1}\right)$ that takes the congruent image of $A$ lying in $\alpha$ to its center in $\alpha$. Then $C_{2}=p(B)$ is an $n$-dimensional cycle in $E_{n}\left(\mathbb{R}^{n+1}\right)$ intersecting the generic fiber at an odd number of points. Consequently, $C_{2}$ is $\mathbb{Z}_{2}$-homologous to $C_{1}$.

As before, the $\mathbb{Z}_{2}$ intersection number of $C_{1}$ and $C_{2}$ in $E_{n}\left(\mathbb{R}^{n+1}\right)$ is nonzero, i.e., in the generic situation they intersect at an odd number of points, which precisely means that the initial body $K$ is contained in an odd number of congruent images of $A_{n+1}$.

Corollary 8 (to the proof). Suppose $A_{n} \subset \mathbb{R}^{n}$ is a centrally and mirror-symmetric $s$-cover bounded by a finite number of regular hypersurfaces. Then each pointed $C B$ field $(K(\alpha), x(\alpha))$ of constant unit width in $\gamma_{n}^{n+1}$ contains a body $K(\alpha)$ that is contained 
in a congruent image of $A_{n}$ centered at $x(\alpha)$. If $K(\alpha)$ is a generic field consisting of smooth convex bodies of constant unit width, then the number of such fibers (and covers) is odd.

Remarks. 1. The $s$-cover $A_{n+1}$ itself satisfies the assumptions of Theorem 5, which allows us to use it for constructing an $s$-cover $A_{n+2} \subset \mathbb{R}^{n+2}$, etc.

2. If we take one of the 1-, 2-, or 3-dimensional covers mentioned above as a "basis" cover, then Theorem 5 yields an infinite series of $s$-covers that are intersections of halfspaces, cylinders, and spheres of unit diameter.

3 . In the text above, we presented polygonal and polyhedral $s$-covers in dimensions $\leq 3$. The author does not know of any polyhedral $s$-covers in dimensions $4,5, \ldots$.

In [9], it was proved that each centrally symmetric 14-hedron $P$ circumscribed about a ball of unit diameter is a universal cover. However, these covers are certainly not rigid, because for each $P$ and each body $K$ of constant unit width in $\mathbb{R}^{4}$ there is a 3 -parametric family of congruent images of $P$ each of which is circumscribed about $K$.

\section{REFERENCES}

[1] V. V. Makeev, The Knaster problem and almost spherical sections, Mat. Sb. 180 (1989), no. 3, 424-431; English transl., Math. USSR-Sb. 66 (1990), no. 2, 431-438. MR0993234 (90d:55005)

[2] __ Affine-inscribed and affine-circumscribed polygons and polyhedra, Zap. Nauchn. Sem. S.Peterburg. Otdel. Mat. Inst. Steklov. (POMI) 231 (1995), 286-298; English transl., J. Math. Sci. 91 (1998), no. 6, 3518-3525. MR.1434300 (98b:52004)

[3] J. Pal, Über ein elementares Variationsproblem, Danske Vid. Selsk. Mat.-Fys. Medd. 3 (1920), no. 2,35 pp.

[4] V. V. Makeev, On affine images of a rhombo-dodecahedron circumscribed about a three-dimensional convex body, Zap. Nauchn. Sem. S.-Peterburg. Otdel. Mat. Inst. Steklov. (POMI) 246 (1997), 191195; English transl., J. Math. Sci. 100 (2000), no. 3, 2307-2309. MR1631812 (99e:52005)

[5] _ Some special configurations of planes that are associated with convex compacta, Zap. Nauchn. Sem. S.-Peterburg. Otdel. Mat. Inst. Steklov. (POMI) 252 (1998), 165-174; English transl., J. Math. Sci. 104 (2001), no. 4, 1358-1363. MR1756722 (2001f:52013)

[6] T. Hausel, E. Makai, and A. Szücs, Polyhedra inscribed and circumscribed to convex bodies, Proceedings of the 3rd International Workshop on Differential Geometry and its Applications and the 1st German-Romanian Seminar on Geometry (Sibiu, 1997), Gen. Math. 5 (1997), 183-190. MR1723608

[7] G. Kuperberg, Circumscribing constant-width bodies with polytopes, New York J. Math. 5 (1999), 91-100; Preprint arXiv: math.MG/9809165. MR.1703205(2000h:52002)

[8] V. V. Makeev, Three-dimensional polytopes inscribed in and circumscribed about compact convex sets, Algebra i Analiz 12 (2000), no. 4, 1-15; English transl., St. Petersburg Math. J. 12 (2001), no. 4, 507-518. MR.1793615 (2001k:52017)

[9] _ Universal coverings. I, Ukrain. Geom. Sb. No. 24 (1981), 70-79. (Russian) MR0629813 (83e:52009)

[10] - On geometric properties of three-dimensional convex bodies, Algebra i Analiz 14 (2002), no. 5, 96-109; English transl., St. Petersburg Math. J. 14 (2003), no. 5, 781-790. MR1970335 (2004c:52001)

[11] - Inscribed and circumscribed polygons of a convex body, Mat. Zametki 55 (1994) no. 4, 128-130; English transl., Math. Notes 55 (1994), no. 3-4, 423-425. MR.1296224|(95h:52002)

Universitetskil Pr. 27, St. Petersburg 190000, Russia

Received 25/DEC/2002

Translated by N. YU. NETSVETAEV 